Original

\title{
Hypospadias and Incomplete Preputial Separation in Male Rats Induced by Prenatal Exposure to an Anti-androgen, Flutamide
}

\author{
Shinsuke Yoshimura ${ }^{1}$, Hajime Yamaguchi ${ }^{1}$, Kazunori Konno ${ }^{1}$, Noriko Ohsawa ${ }^{1}$, \\ Satoshi Noguchi ${ }^{1}$, and Akiko Chisaka ${ }^{1}$ \\ ${ }^{1}$ Hatano Research Institute, Food and Drug Safety Center, 729-5, Ochiai, Hadano, Kanagawa 257-8523, Japan
}

\begin{abstract}
Hypospadias was induced in male Sprague-Dawley rats by prenatal exposure to $30 \mathrm{mg} / \mathrm{kg} /$ day of flutamide from gestational days 14 to 17 , or from 18 to 21 . Their external genitalia were examined histopathologically and compared to untreated controls. On postnatal day 6 , the glans penis of untreated controls was bordered with epithelium. On postnatal day 22, papillae were recognized on the glans penis side, and cornification started close to the tip of the papilla on postnatal day 35. On postnatal day 42 cornification spread to the surface of the glans penis. The cornification progressed from tip to base of the glans penis, and from dorsal to ventral. When cornification reached the base of the glans penis, separation of the double layered epithelia was complete and the animal was considered sexually mature. Flutamide treatment on gestational days 14-17 induced a defect in the ventral half of the glans penis (cleft phallus) and cleft in the ventral prepuce (cleft prepuce) in the male pups, while treatment on gestational days 18-21 induced cleft phallus without apparent abnormalities in the prepuce. The external urethral orifice opened at the ventral end of the glans penis (hypospadias) in both treatment groups. In male pups with cleft phallus, cornification of the dorsal epithelium followed by separation of the prepuce occurred, while separation of the ventral part of glans penis did not occur because epithelium was not formed at the ventral part of the glans penis. Consequently, the onset of puberty was not decided in these animals. These findings indicate that the defect of the ventral half of the phallus is the reason why the time of sexual maturation was not decided, and that there is a difference between the phallus and prepuce in the sensitive period concerning the development of flutamide-induced malformations.
\end{abstract}

(J Toxicol Pathol 2004; 17: 113-118)

Key words: flutamide, anti-androgen, rat, prenatal exposure, hypospadias

\section{Introduction}

Preputial separation, which is observed as separation of the prepuce from the glans penis, has been used as a sign of puberty in the male rat. Histological observation on the progress of preputial separation after cornification at the lining of prepuce and surface of glans penis was well described using Long-Evans rats in $1942^{1}$. Preputial separation is thought to be dependent on androgens, because castration blocked preputial separation, and the addition of testosterone (TS) or dihydrotestosterone (DHT) recovered the effect of castration ${ }^{1,2}$. In recent years, preputial separation has been used as an endpoint to evaluate endocrine disrupting chemicals. Although the observation

Received: 26 January 2004, Accepted: 15 April 2004

Mailing address: Shinsuke Yoshimura, Laboratory of Toxicology, Hatano Research Institute, Food and Drug Safety Center, 729-5,

Ochiai, Hadano, Kanagawa 257-8523, Japan

TEL: 81-463-82-4751 FAX: 81-463-82-9627

E-mail: yoshimura.s@fdsc.or.jp of preputial separation is a useful tool for detecting sexual maturation, anti-androgenic chemicals induce hypospadias in male rats by intrauterine exposure. The time of sexual maturation is determined by complete separation of the prepuce from the ventral surface of the glans penis, but in males with hypospadias, puberty is undetermined because this complete separation in the glans penis is not evident ${ }^{3}$.

The purpose of this study was to reveal the histological process of normal and abnormal preputial separation, as well to reveal the reason why the time of sexual maturation cannot be decided in males with hypospadias induced by prenatal exposure to flutamide (FLU), an anti-androgenic chemical, in Sprague-Dawley rats. FLU was administered on gestational days (GD) 14-17 (expected to be the most sensitive period for hypospadias) or on GD 18-21 (thought to be a less sensitive period for hypospadias).

\section{Materials and Methods}

Sprague-Dawley rats (Crj:CD (SD) IGS), 30 males and 33 females, 11 weeks of age, were obtained from Charles 
River Japan, Inc. (Atsugi, Japan). All animals were acclimatized to laboratory conditions and quarantined for one week before mating. Rats used for this study were selected based upon general condition, appearance and behavior during the acclimatization period. Animals were housed individually in wire-bottom metal cages $(220 \times 270$ $\times 190 \mathrm{~mm}$ ) and kept in a barrier sustained animal room that was maintained at $21.0-25.0^{\circ} \mathrm{C}$ and $40.0-75.0 \%$ relative humidity with a 12-hour artificial light cycle (lighting from 7:00 to 19:00). Fifteen changes of room air per hour were provided. Commercial diet CE-2 (CLEA Japan, Inc., Tokyo) and water (Hadano City) were available ad libitum throughout the study. The protocol of the present study was approved by the Animal Use Committee of the Hatano Research Institute.

The untreated control group consisted of 18 females. While the second group, consisting of 9 females, was treated orally with $30 \mathrm{mg} / \mathrm{kg} /$ day of FLU (Sigma Chemical Co., St. Louis, USA) dissolved in corn oil (Nacalai Tesque, Inc., Kyoto, Japan) from GD 14 to 17 , the third group, consisting of 6 females, was treated with the same dose from GD 18 to 21. From the result of a preliminary study the dosage was decided as $30 \mathrm{mg} / \mathrm{kg} /$ day, because pregnant rats died after administration of $100 \mathrm{mg} / \mathrm{kg} /$ day of FLU. To obtain pregnant animals, 12-week-old females were cohabited overnight on a 1:1 basis with males 12 weeks of age or older. Females were considered to be at GD 0 when daily examination revealed a vaginal plug. All pregnant animals were housed in cages with animal bedding (PAPER CLEAN $^{\circledR}$, Japan SLC, Inc., Shizuoka) from GD 18 until postpartum day 10, and allowed to give birth. On postnatal day (PND) 6 (PND 0 is the day of delivery) all female pups were discarded. Body weights of male pups were measured on PND 0, 6, 22, 35 and 56. Progress of preputial separation of male pups was observed macroscopically from PND 35.

Control male pups were sacrificed by exsanguination under anesthesia on PND 6, 22, 35, 42 and 56 (number of pups in group 1 were $6,16,15,4$ and 24 , respectively). Male pups from FLU-treated females were sacrificed under anesthesia on PND 6 and 56 (number of pups in group 2 were 18 and 41, respectively, and those in group 3 were 8 and 21 , respectively). After macroscopic examination, the prepuce and penis were dissected and fixed with $0.1 \mathrm{~mol} / \mathrm{L}$ phosphate buffered $10 \%$ formalin solution. Sagittal slices of the prepuce and penis were embedded in paraffin, and sections were stained with hematoxylin-eosin ( $\mathrm{H} \& \mathrm{E})$ for histopathological examination.

\section{Results}

On macroscopic examination, the glans penis of control males was covered with prepuce, and the prepuce could be completely retracted to expose the glans penis until PND 46 (Figs. 1A, 1B). Prepuce of males prenatally exposed to FLU on GD 14-17 had a cleft at the ventral part (cleft prepuce), and the glans penis was observed from the cleft (Fig. 1C). The ventral part of the glans penis of these males was incompletely formed (cleft phallus) and the os penis was often exposed (Fig. 1D). The incidence of cleft prepuce was $80 \%(33 / 41)$, and the incidence of cleft phallus was $90 \%$ (37/ 41). Cleft prepuce is usually observed with cleft phallus. Another 4 males showed no cleft on their prepuce or phallus, while preputial separation was delayed or incomplete on PND 56. Although there was no cleft at the prepuce of males exposed to FLU on GD 18-21 (Fig. 1E), the ventral part of the glans penis was incompletely formed (cleft phallus, Fig. $1 \mathrm{~F}$ ), and incidence of the cleft phallus in this group was $100 \%(21 / 21)$. Body weight gains of males were not affected by FLU exposure.

Upon histological examination of untreated controls on PND 6, the glans penis was bordered with specific epithelium (Fig. 2A). The epithelium consisted of outer and inner basal layers (Fig. 2B). The outer layer lined the inside of the prepuce and the inner layer covered the glans penis. The urethra was located in the center of the glans penis and the os penis was observed between the dorsal surface of the glans penis and the urethra (Fig. 2A). On PND 22, there were many papillary processes from the glans penis (arrows in Fig. 2C), and the surface of these processes was covered with squamous epithelial cells. At this point the two basal layers lost their parallel arrangement. On PND 35, the epithelial layer consisted of stratified squamous epithelium, and the surface of the papillary processes (arrows in Fig. 2D) was covered with cornified cells. The cornified layer was limited to the surface of these processes. On PND 42, epithelial cells between the papillae also cornified, and both surfaces of the penis and prepuce consisted of keratinized stratified squamous epithelium. Cornification and separation were incomplete at the basal part of the glans penis. Separation at the ventral surface of the glans penis was more delayed than that at the dorsal surface. On PND 56 cornification was complete from the tip to the base of the glans penis and the ventral surface also showed cornified layers (Figs. 2E, 2F). The preputial separation was complete across the entire surface of the glans penis.

Histological examination of males prenatally exposed to FLU on GD 14-17 revealed a cleft at the ventral surface of genital tubercle on PND 6 (arrow in Fig. 3A). The urethra was not located in the center of the glans penis, but instead was observed at the ventral surface of the glans penis. The dorsal part of the glans penis was bordered by epithelium as observed in controls, while the ventral part was covered with urethral epithelium. This finding indicates that the ventral half of glans penis was not formed (comparison with controls as shown by an asterisk (*) in Fig. 2A). The cavernous body of the penis was tortuous and also observed in PND 56 males exposed to FLU on GD 14-17 (Fig. 3B). The dorsal surface of the glans penis and prepuce of PND 56 males were covered with keratinized stratified squamous epithelium, and the prepuce was separated from the glans penis (Fig. 3B). The ventral part of the glans penis and ventral epithelium were not formed between the urethra and subcutis, the ventral surface of the glans penis was not covered with squamous epithelium and the preputial 


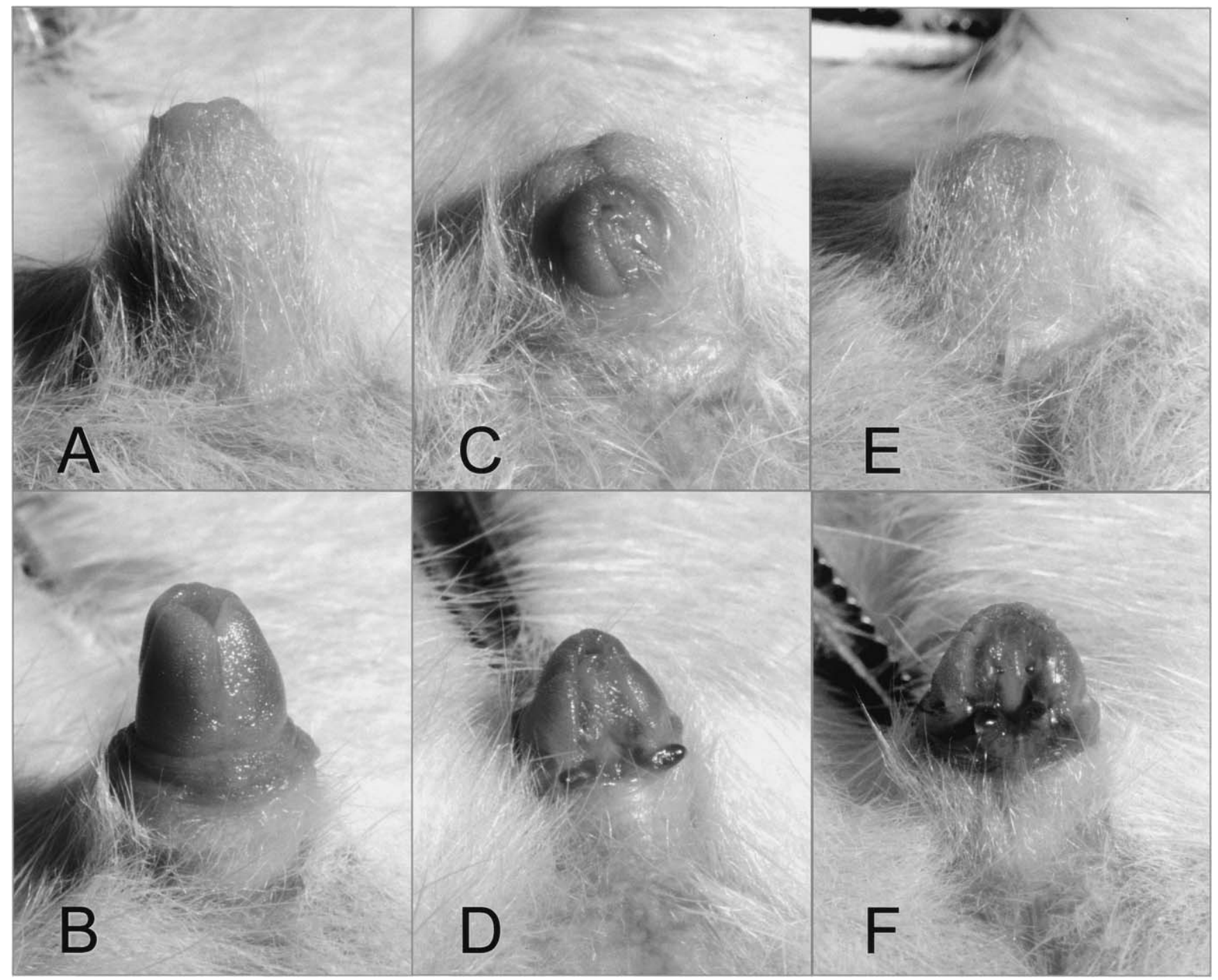

Fig. 1. Ventral surface of genital tubercle (A, C and E) and glans penis (B, D and F) of males at PND 56. A and B: Control rat. Prepuce is completely retracted. C and D: Male rat prenatally exposed to FLU on GD 14-17. Ventral side of the prepuce has a cleft, and the glans penis is observed from the cleft. Ventral part of the glans penis is incompletely formed (cleft phallus) and os penis is observed. E and F: Male rat prenatally exposed to FLU on GD 18-21. Prepuce does not have a cleft at the ventral side. Glans penis shows cleft phallus and os penis is observed.

separation did not progress at the ventral part. The external urethral orifice opened at the ventral surface of the glans penis (hypospadias). The preputial tissue was hypoplastic and the tip of the penis was not overlain with prepuce.

The glans penis of PND 6 males exposed to FLU on GD 18-21 was covered with skin, and a cleft was not observed at the ventral part of genital tubercle (Fig. 3C). The dorsal part of the glans penis was bordered by epithelium, but the ventral part of the glans penis and ventral epithelium were not formed between the urethra and subcutis (comparison with controls as shown by an asterisk $(*)$ in Fig. 2A). The tortuous structure of the cavernous body was indistinct. The prepuce overlaid the glans penis of PND 56 males exposed on GD 18-21 (Fig. 3D). The dorsal surface of the glans penis of these males was covered with keratinized stratified squamous epithelium, and the prepuce was separated from the glans penis. The ventral part of the glans penis and ventral epithelium were not formed between the urethra and subcutis, and preputial separation did not progress at the ventral part. In these rats the external urethral orifice opened at the ventral surface of the glans penis.

\section{Discussion}

As described above, preputial separation in untreated rats initiated from cornification of the epithelium on the penile side lying in the dual phasic epithelium between the glans penis and prepuce. Cornification began at the surface very close to the apex of the papillary process from the glans penis, and when the cornification reached the next papilla the prepuce separated from the glans penis. Preputial separation progressed from the tip of the glans penis towards its base, 


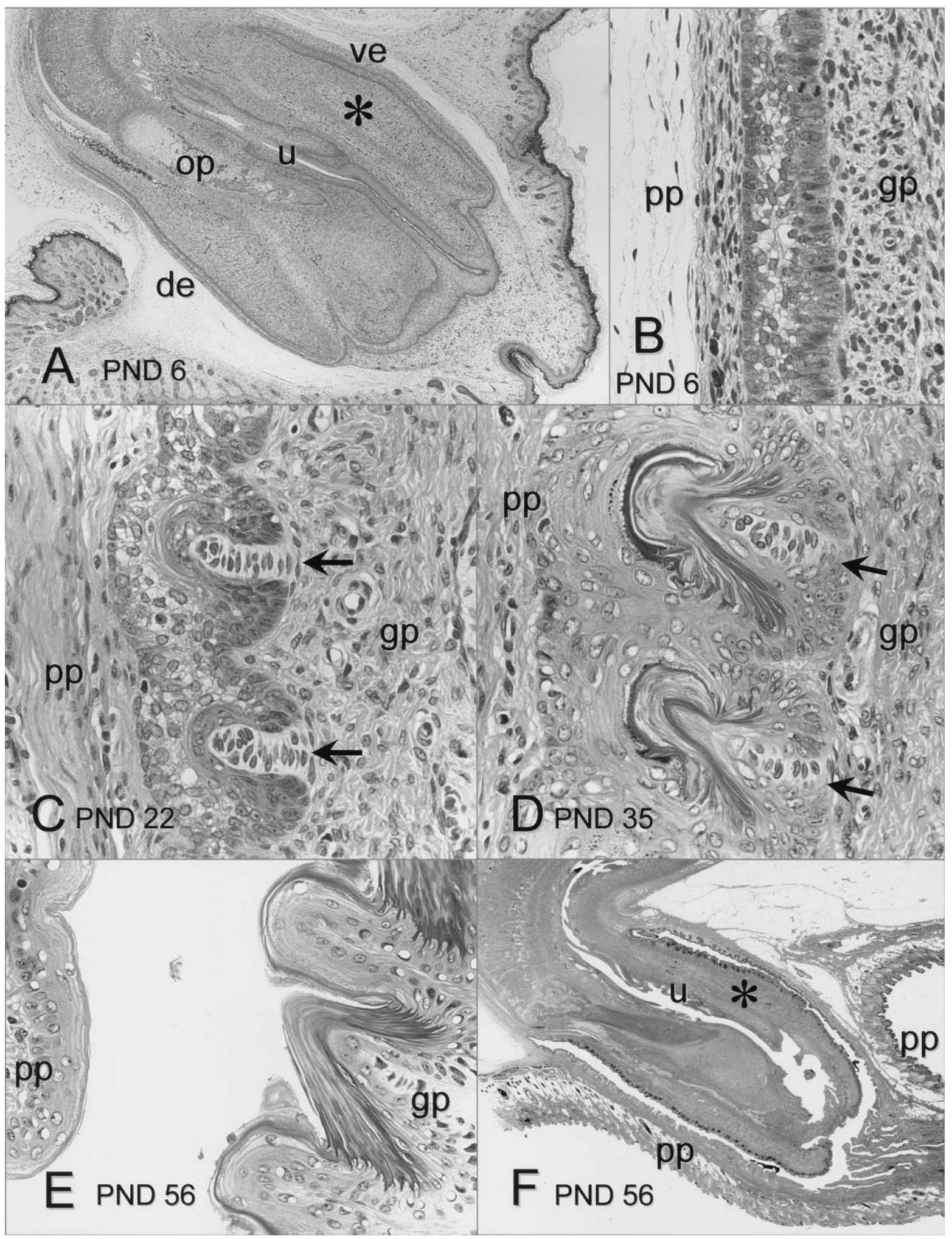

Fig. 2. Sagittal sections of the genital tubercle from control males.

A: Genital tubercle of a male on PND 6. Glans penis is bordered with dorsal epithelium (de) and ventral epithelium (ve). Urethra (u) is located in the center of the glans penis. op: os penis. *: ventral half of the glans penis.

B, C, D and E: Epithelium between the dorsal part of the glans penis (gp) and prepuce (pp) on PND 6, 22, 35 and 56, respectively. Epithelium of PND 6 consisted of outer and inner basal layers (B). Squamous epithelial cell of PND 22 is covering the papillary processes (arrows) from the glans penis (C). Epithelial layer of PND 35 consists of stratified squamous epithelium, and cornified layer is covering the papillary processes (arrows in D). Whole surface of both the glans penis and prepuce is covered with cornified layer on PND 56 (E). F: Glans penis and prepuce on PND 56. Preputial separation is completed. Urethra $(\mathrm{u})$ is located in the center of the glans penis.

*: ventral half of the glans penis. H \& E. Magnification, A: $\times 35, \mathrm{~B}, \mathrm{C}$ and $\mathrm{D}: \times 400, \mathrm{E}: \times 330, \mathrm{~F}: \times 9$. 


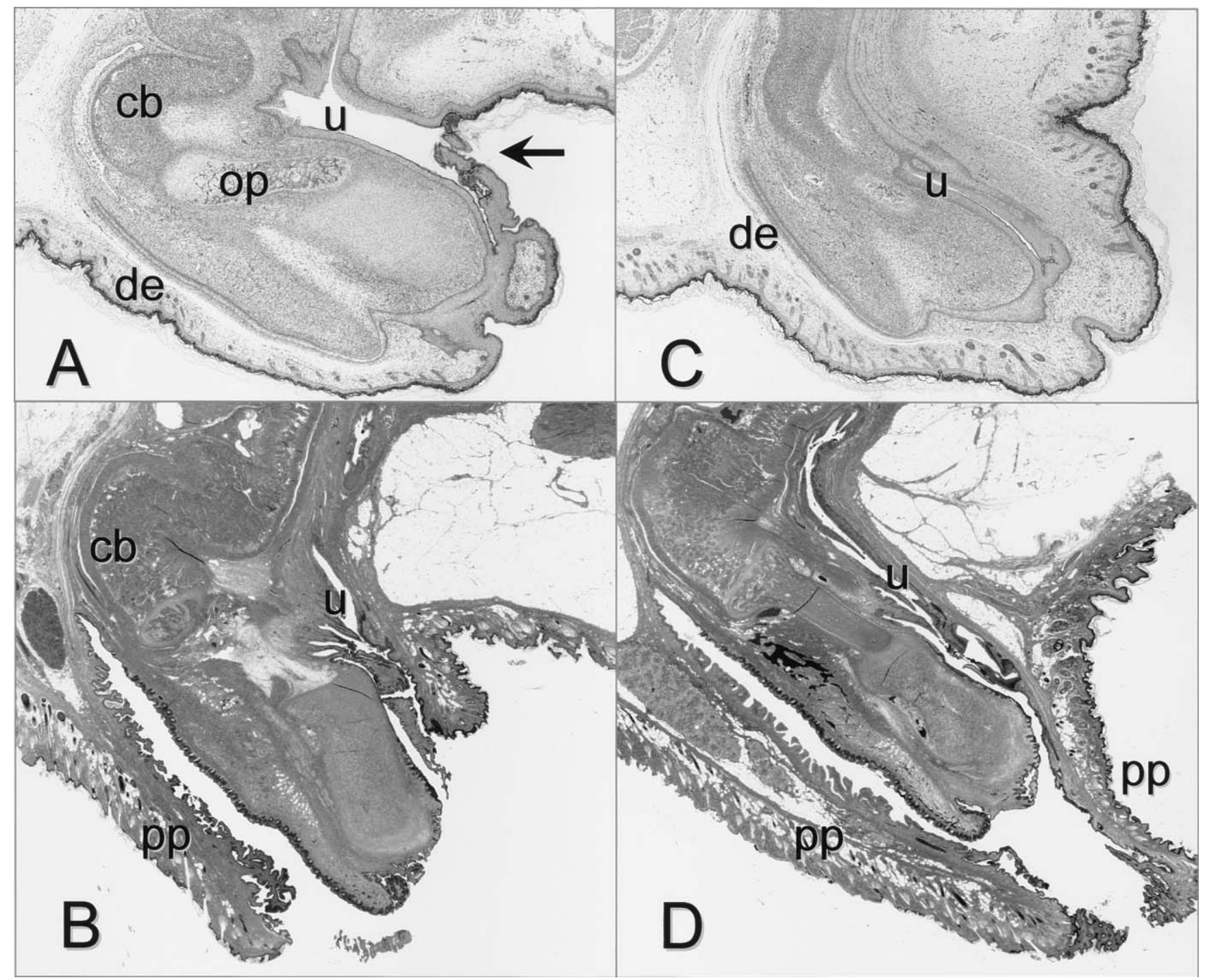

Fig. 3. Sagittal sections of genital tubercle from males prenatally exposed to FLU on GD 14-17 (A, B) or GD 18-21 (C, D), sacrificed on PND 6 $(\mathrm{A}, \mathrm{C})$ and PND 56 (B, D).

A: There is a cleft (arrow in the figure) at the ventral side of genital tubercle. Urethra (u) is observed at the ventral surface of the glans penis. Dorsal part of the glans penis is bordered with epithelium (de). Cavernous body (cb) shows tortuous structure.

B: Prepuce (pp) is separated from the glans penis at the dorsal part. Prepuce is hypoplastic, and the glans penis is not completely overlain with the prepuce. Urethra is located between the glans penis and subcutis.

$\mathrm{C}$ : Cleft is not formed at the ventral side of genital tubercle. Dorsal part of the glans penis is bordered with epithelium (de). Ventral part of the glans penis and ventral epithelium is not formed. Urethra is located between the glans penis and subcutis.

D: Prepuce is separated from the glans penis at the dorsal part. The ventral part of the glans penis and ventral epithelial layer is not formed. The glans penis is completely overlain with the prepuce.

H\&E. Magnification, A and C: $\times 27$; B and D: $\times 10$.

and also from the dorsal to ventral surface of the glans penis Histological features observed in controls of this study were almost the same as shown in Long-Evans rats ${ }^{1}$. Complete separation was not observed in animals exposed to FLU in their fetal period, since they had a cleft phallus at their ventral surface of the glans penis. Histopathological examination revealed defects in the ventral part of the glans penis and lack of an epithelial layer at the ventral part in newborn rats.

Induction of hypospadias has been reportedly caused by various chemicals, which include anti-androgens such as FLU, vinclozolin and finasteride. FLU is a well-known potent androgen receptor antagonist and is used as a nonsteroidal anti-androgen drug for the treatment of prostate cancer. FLU inhibits TS and DHT binding to the intracellular androgen receptor and prenatal/perinatal exposure to FLU induces abnormalities in the genital tract such as hypospadias, agenesis of the prostate, epididymis and vas deferens ${ }^{2,4,5}$. Vinclozolin, a fungicide, is also an androgen receptor antagonist, and induces hypospadias in 
rats by perinatal (GD 14 to day 3 postpartum) ${ }^{6}$ or prenatal (for 2 days in GD 12-21) ${ }^{7}$ administration. Finasteride, which inhibits $5 \alpha$-reductase conversion of TS to DHT, also induces hypospadias in male rats exposed from GD 15 to day 21 postpartum, and based on this finding DHT is thought to be involved in the development of external genitalia ${ }^{8}$. While the Wolffian ducts are dependent on TS, their derivatives such as the epididymis, vas deferens or seminal vesicles were not affected by intrauterine exposure to finasteride ${ }^{8}$. Hypoplastic change in the genital tubercle was reported in fetuses exposed to finasteride from GD 6 to $20^{9}$. Wedge shaped mesenchymal tissue between rectum and urogenital sinus failed to develop in these fetuses, and the urethra opened near the base of the tubercle. This finding indicates that the mesenchymal wedge may be the most sensitive area to loss of the effect of DHT in male fetuses. Our study also revealed a defect in the ventral part of the glans penis, in which preputial separation could not progress, and this is the reason why the time of sexual maturation could not be decided in males with hypospadias.

Androgen receptors are detectable in the mesenchymal cells of the rat urogenital tubercle from fetal day 14 onwards ${ }^{10}$. In many studies of sexual differentiation and male reproductive organ malformation, dosing starts from GD 12 or 14 . The most sensitive period to induce hypospadias is reportedly GD 15-16 with $400 \mathrm{mg} / \mathrm{kg}$ of vinclozolin exposure, and the incidence was $42 \%$ (10/24), while only weak sensitivity $(11 \%, 1 / 9)$ was found with treatment on GD $17-18^{7}$. Similar results were obtained in our study of vinclozolin (unpublished data) ${ }^{11}$. Exposure to $100 \mathrm{mg} / \mathrm{kg}$ of vinclozolin on GD 14-17 induced cleft phallus with cleft prepuce, and the incidence was $85 \%$, but exposure on GD 18-21 induced no abnormalities in external genitalia. Pregnant females or newborn pups died after exposure to $200 \mathrm{mg} / \mathrm{kg}$ of vinclozolin. Finasteride exposed rats also showed similar results ${ }^{8}$. Male pups exposed to $20 \mathrm{mg} / \mathrm{kg}$ of finasteride on GD 16-17 showed hypospadias, and the incidence was $39 \%(14 / 36)$, while incidence in the GD 18 19 group was $0 \%(0 / 36)$.

Many reports describe the malformation of the phallus as hypospadias, but details and the incidence of the prepuce malformations are not clear. In our study, pregnant rats were administered with $30 \mathrm{mg} / \mathrm{kg}$ of FLU on GD 14-17, which was considered to be the sensitive period, and the incidence of cleft phallus and cleft prepuce was compared to the exposure on GD 18-21. Male pups exposed to FLU on GD 14-17 showed cleft phallus with hypospadias (90\%) and cleft prepuce (80\%), while males exposed on GD 18-21 had cleft phallus with hypospadias $(100 \%)$ without apparent abnormality in the prepuce. A lower dose of FLU also showed similar results with lower incidence (unpublished data $)^{11}$. Exposure of $10 \mathrm{mg} / \mathrm{kg}$ of FLU on GD 14-17 induced cleft phallus (58\%) and cleft prepuce $(25 \%)$, while exposure on GD 18-21 induced cleft phallus (25\%) without apparent abnormality in the prepuce. These findings show that the period of sensitivity to FLU in terms of phallus malformation is different from vinclozolin and finasteride, and also that there are differences among the sensitive periods between the phallus and prepuce concerning FLUinduced malformations.

Acknowledgments: This work was supported by Health and Labour Sciences Research Grants from the Ministry of Health, Labour and Welfare of Japan. We would also like to thank Dr. Masayoshi Kanisawa for proofreading this manuscript.

\section{References}

1. Lyons WR, Berlin I, and Friedlander S. Cornification of balano-preputial epithelium in normal rats and in castrated rats treated with testosterone propionate. Endocrinology 1942; 31: 659-663.

2. Korenbrot CC, Huhtaniemi IT, and Weiner RI. Preputial separation as an external sign of pubertal development in the male rat. Biol Reprod 1977; 17: 298-303.

3. McIntyre BS, Barlow NJ, and Foster PMD. Androgenmediated development in male rat offspring exposed to flutamide in utero: permanence and correlation of early postnatal changes in anogenital distance and nipple retention with malformations in androgen-dependent tissues. Toxicol Sci 2001; 62: 236-249.

4. Mylchreest E, Sar M, Cattley RC, and Foster PMD. Disruption of androgen-regulated male reproductive development by di( $n$-butyl) phthalate during late gestation in rats is different from flutamide. Toxicol Appl Pharmacol 1999; 156: 81-95.

5. Miyata K, Yabushita S, Sukata T, Sano M, Yoshino H, Nakanishi T, Okuno Y, and Matsuo M. Effects of perinatal exposure to flutamide on sex hormones and androgendependent organs in F1 male rats. J Toxicol Sci 2002; 27: 19-33.

6. Gray LE Jr, Ostby JS, and Kelce WR. Developmental effects of an environmental antiandrogen: the fungicide vinclozolin alters sex differentiation of the male rat. Toxicol Appl Pharmacol 1994; 129: 46-52.

7. Wolf CJ, LeBlanc GA, Ostby JS, and Gray LE Jr. Characterization of the period of sensitivity of fetal male sexual development to vinclozolin. Toxicol Sci 2000; 55: 152-161.

8. Clark RL, Anderson CA, Prahalada S, Robertson RT, Lochry EA, Leonard YM, Stevens JL, and Hoberman AM. Critical developmental periods for effects on male rat genitalia induced by finasteride, a $5 \alpha$-reductase inhibitor. Toxicol Appl Pharmacol 1993; 119: 34-40.

9. Anderson CA and Clark RL. External genitalia of the rat: normal development and the histogenesis of $5 \alpha$-reductase inhibitor-induced abnormalities. Teratology 1990; 42: 483496.

10. Bentvelsen FM, Brinkmann AO, van der Schoot P, van der Linden JETM, van der Kwast TH, Boersma WJA, Schröder FH, and Nijman JM. Developmental pattern and regulation by androgens of androgen receptor expression in the urogenital tract of the rat. Mol Cell Endocrinol 1995; 113: 245-253.

11. Yoshimura S. Effects of prenatal or postnatal exposure to chemicals on preputial separation of male rats (unpublished). 